\title{
Multi-user Opportunistic Scheduling using Power Controlled Hierarchical Constellations*
}

\author{
Md. Jahangir Hossain ${ }^{1}$, Mohamed-Slim Alouini ${ }^{2}$, and Vijay K. Bhargava ${ }^{1}$ \\ Department of Electrical and Computer Engineering \\ ${ }^{1}$ University of British Columbia, Vancouver, BC, Canada \\ ${ }^{2}$ University of Minnesota, Minneapolis, MN, USA \\ E-mail: <\{jahangir, vijayb\}@ece.ubc.ca $>$
}

\begin{abstract}
In this paper, we study an application of hierarchical constellations (known also as embedded, multi-resolution, or asymmetrical constellations) for multi-user opportunistic scheduling. The key idea is to rely on hierarchical constellations to transmit information to two or more best users simultaneously in each transmission. The transmit power as well as the constellation parameters is changed according to the link qualities of the selected users in a way that a given target bit error rate (BER) is satisfied. We present the expressions for the average transmit power, the outage probability and the packet transmission probability with truncated channel inversion power control. Numerical examples show that, in an identical and independent fading condition, the proposed hierarchical constellation-based multi-user scheduling scheme offers higher frequency of channel access to the users at the expense of average transmission power compared to the classical opportunistic single user scheduling. The required additional power decreases as the number of users increases in the system. We also compare our proposed hierarchical constellation-based multi-user scheduling scheme with a uniform constellation-based time-slotted multi-user opportunistic scheduler. This comparison shows that although this time slotted system offers the same access probability as the hierarchical scheme, it requires more average transmit power than the newly proposed hierarchical scheme.
\end{abstract}

\section{INTRODUCTION}

In opportunistic scheduling (also known as multi-user diversity) (see, for example, [1] and the references therein), the scheduler at the base station selects a single "best" user in each time slot based on available channel state information and transmits to this user. Although this single best user opportunistic scheduling offers overall throughput gain, the frequency of channel access of a given user can be quite low, especially with large number of users in the system. As a consequence, the delay experienced by a user to get service can be high and also the packet loss due to buffer over flow for a memory limited system can be quite high. This motivates the design of opportunistic scheduling schemes which can take advantage of channel variations among the users to provide higher throughput while offering more frequent channel access and packet transmission to the users.

\footnotetext{
*This work is supported in part by the University British Columbia graduate fellowship: Hui memorial fellowship, in part by the Natural Sciences and Engineering Council of Canada (NSERC), Canada, and in part by the Center of Transportation Studies (CTS) through the Intelligent Transportation Systems (ITS) Institute, Minneapolis, Minnesota, USA
}

Recently, the concept of two best user scheduling, which assigns different number of orthogonal codes to two best users in each transmission, has been proposed for code division multiple access (CDMA) systems [2], [3].

Hierarchical constellations consist of non-uniformly spaced signal points (see, for example, [4], [5]) and thus offer different levels of protection (i.e., bit error rate (BER)) to the transmitted bits in a message symbol according to their priorities. Due to this capability of providing different levels of protection to the bits in the same message symbol, this type of constellations received a great deal of attention for many applications. Examples of these applications include, multimedia transmission [7] and downlink multiplexing [6] and simultaneous voice and multi-class data transmission [8]. In our previous work [6], we proposed a hierarchical scheme for downlink multiplexing which multiplexes information bits for different users in each symbol transmitted from the adaptive hierarchical modulator. It is shown that the proposed hierarchical scheme requires less power than a classical time division multiplexing that employs uniform constellations. The power gain came from the superposition of bit strings in hierarchical fashion according to their priorities [10]. Similar idea of superposing bits from different users using hierarchical constellations was proposed for orthogonal frequency division multiple access (OFDMA) system [9]. This scheme leads to sharing a given OFDM sub-carrier between two or more users having better channel qualities in that sub-carrier. Again it was was shown that the sharing a sub-carrier between two or more users requires less transmit power than timedivision multiple access OFDM (TDMA-OFDM) and TDMA frequency division multiple access technique (TDMA-FDMA) systems.

These power efficient hierarchical multiplexing schemes motivates us to study an application of hierarchical constellations in multi-user opportunistic scheduling which provides access information to multiple best users rather than a single best user in a given time slot. Contrary to the orthogonal code allocation-based two best user scheduling schemes [2], [3], in this letter we propose a modulation assisted multiple best user opportunistic scheduling scheme. The detailed description of our proposed scheme will be given in later section. We present expressions for the average transmit power, the outage probability and the packet transmission probability 
with truncated channel inversion power control [11] for a fading environment where users' channels varies identically and independently (i.i.d.) ${ }^{1}$. Using these expressions, we explore the trade-offs between the access probability and the power efficiency of multi-user opportunistic scheduling using hierarchical constellations. The numerical examples show that, in an i.i.d fading environment, the multi-user scheduling using hierarchical constellation offers more frequent channel access to the users at the expense of an increase in average transmission power compared to the classical opportunistic singleuser scheduling. This required additional power decreases to a small value as the number of users in the system increases. For example, in a two best users scheduling scenario, the access probability is doubled with $0.8 \mathrm{~dB}$ of power increment for 30 users in the system. We also compare the hierarchical multi-user scheduling scheme with a time-slotted multi-user opportunistic scheduling. In this time-slotted scheme two or more best users are scheduled for transmission in a mini time-slotted fashion. The comparison shows that although the uniform constellation based time slotted scheme offers the same access probability as the hierarchical scheme, it requires more average transmit power than our proposed hierarchical scheme.

\section{Multi-USER Opportunistic SCHEdUling}

\section{A. Hierarchical Constellations}

Throughout this paper, we consider two best user opportunistic scheduling using hierarchical 4/16-QAM constellation which is constructed with two levels of hierarchy. Scheduling more than two best users using higher order hierarchical modulation is quite straightforward. The detailed description of generalized hierarchical constellations can be found, for example, in [4].

A hierarchical 4/16-QAM constellation (see, for example, [5], [12]), as shown in Fig. 1, can be modeled as follows. We assume that there are two streams of data. Each one of these incoming streams carries information of a particular priority. For every channel access two bits are chosen from each level of priority. The two bits from the highest priority stream are assigned to the most significant bit (MSB) position in the inphase (I) and quadrature (Q) channels. Consequently, the two bits from the least priority stream are assigned to the least significant bit (LSB) position in the I and Q channels. In Fig. 1, the fictitious 4-QAM constellation denoted by the black circle represents the highest priority (hereafter first hierarchy $\mathrm{H}_{1}$ ). The distance $d_{1}$ between the fictitious symbols represents the first priority. The actual transmitted white symbols represent the 16-QAM constellation. This is the second level of hierarchy (hereafter second hierarchy $\mathrm{H}_{2}$ ) and the distance $d_{2}$ between the actual transmitted symbols represents the second priority.

\footnotetext{
${ }^{1}$ Although the expressions have been derived for an i.i.d fading environment, our proposed scheme can be used for a non-i.i.d. fading environment and similar expressions can be evaluated for a non-i.i.d. environment using the same methodology.
}

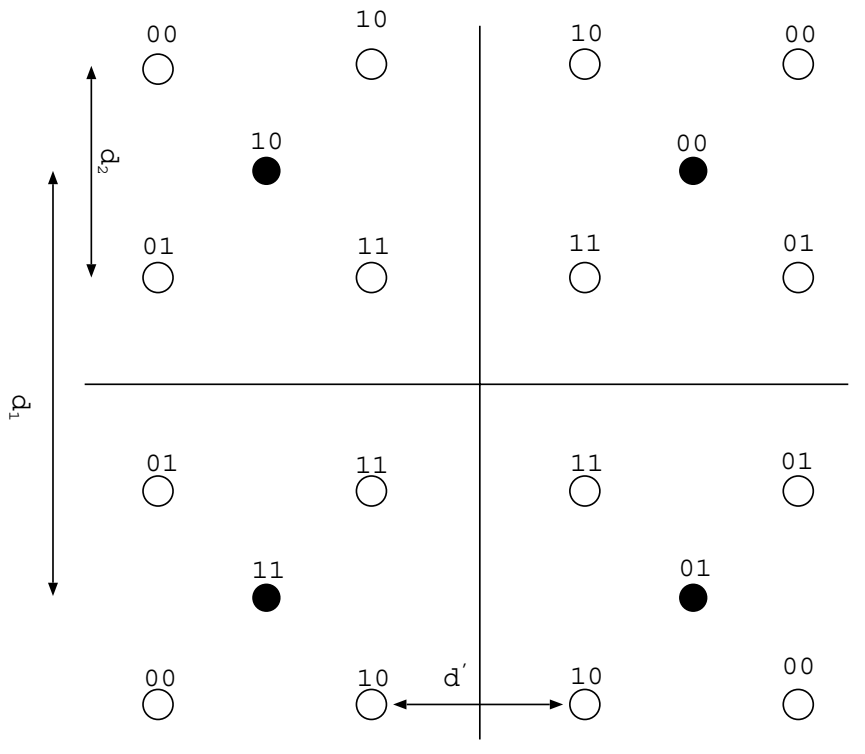

Fig. 1. Hierarchical 4/16-QAM constellation.

The exact bit error rate (BER) expressions over additive white Gaussian noise (AWGN) channel for this hierarchical 4/16-QAM constellation were derived in [12]. The BER equations in [12] are not invertible in terms of transmit power and the constellation priority parameter for given other parameters. However, the well known SNR-gap approximation [13] leads to the ease inversion for the transmit power and a simple design of hierarchical constellations (see [9]). We use the same approximation to evaluate down the average transmit power analytically. According to the SNR-gap approximation, the average power of $k$ th hierarchy is given by [9], [13]

$$
P_{k}=\frac{d_{k}^{2}\left(2^{m}-1\right)}{6}
$$

where $d_{k}$ is the distance between the constellation symbols in the $k$ th hierarchy and $m$ is the number of bits assigned to $k$ th hierarchy which is equal to two in the case of hierarchical 4/16-QAM. The distances $d_{k}(k=1,2)$ as shown in Fig. 1 are calculated recursively as follows. The distance between the actual constellation (16-QAM) symbols, $d_{2}$ is calculated as

$$
d_{2}=\sqrt{6 \times \frac{2 N_{0} \Gamma}{\alpha^{2}}}
$$

where $\alpha^{2}$ is the channel fading gain of the user assigned to second hierarchy, $N_{0}$ is the noise power spectral density, and $\Gamma$ is the SNR-gap of the uncoded QAM constellation and given by

$$
\Gamma=\frac{1}{3}\left[\mathrm{Q}^{-1}\left(\frac{\mathrm{BER}_{0}}{4}\right)\right]^{2}
$$

where $\mathrm{Q}^{-1}(\cdot)$ is the inverse standard Gaussian Q-function and $\mathrm{BER}_{0}$ is the target BER. The distance between the actual symbols in different quadrant $d^{\prime}$ as shown in Fig. 1, can be 
calculated as

$$
d^{\prime}=\sqrt{6 \times \frac{2 N_{0} \Gamma}{\beta^{2}}}
$$

where $\beta^{2}$ is the fading gain of the user assigned to the first hierarchy. Now the distance between fictitious symbols (4QAM) in the first hierarchy can be written as (see Fig. 1)

$$
d_{1}=d^{\prime}+d_{2}
$$

Using Eqs. (1)-(5), the total transmit power with hierarchical 4/16-QAM constellation (assuming that the second hierarchy is assigned to a user with channel fading gain $\alpha^{2}$ and that the first hierarchy is assigned to a user with channel gain $\beta^{2}$ ) can be expressed as

$$
P(\alpha, \beta)=\frac{C_{2}}{\alpha^{2}}+\frac{C_{2}}{\alpha^{2} \beta^{2}}\left(\alpha^{2}+2 \alpha \beta+\beta^{2}\right)
$$

where the constant $C_{2}=2 N_{0} \Gamma\left(2^{2}-1\right)$.

\section{B. Hierarchical Constellation-based Multi-user Opportunistic Scheduling}

To describe our proposed multi-user opportunistic scheduling let us consider a base station (BS) is transmitting to $K$ users opportunistically. The users have to meet a predetermined target $\mathrm{BER}, \mathrm{BER}_{0}$ and the channel variation among the users is assumed to be i.i.d. The channel fading amplitude of a given user is Rayleigh faded which corresponds to the following probability density function (pdf) for the channel fading gain $g$

$$
p_{G}(g)=\frac{1}{g_{0}} \exp \left(-\frac{g}{g_{0}}\right)
$$

where $g_{0}$ is the average channel gain. The other channel models, such as, Nakagami fading, can also be used in our proposed framework. Assuming that the received channel gain of each user is known to the BS scheduler,

we now describe the scheduling as well as the corresponding adaptation process.

- Step 1: Based on users' channel qualities, the scheduler at the BS ranks the users and picks up the first (denoted by $\mathrm{U}_{1}$ ) and the second best user (denoted by $\mathrm{U}_{2}$ ).

- Step 2: If the channel fading gain of the second best user, $g_{2}$ is above a threshold $g_{\mathrm{th}}^{2}$, go to the next step. Otherwise declare a transmission outage for that time step and restart from step 1 in the next time slot.

- Step 3: Since the user, $\mathrm{U}_{1}$ requires less protection than user $\mathrm{U}_{2}$, hierarchy $\mathrm{H}_{2}$ is assigned to the user $\mathrm{U}_{1}$ whereas hierarchy $\mathrm{H}_{1}$ is assigned to the user $\mathrm{U}_{2}$. With this assignment, the transmitter adapts the transmission power as well as the relative distances between the symbols $\left(d_{1}, d_{2}\right.$ and $\left.d^{\prime}\right)$ such as the target BER, BER ${ }_{0}$ is met with the corresponding channel fading gains $g_{1}$ and $g_{2}$.

The position of the bits in a transmitted symbol for the selected users are sent via a feed-forward channel. Thus the selected users receive the symbols and look only for the bits in particular positions within the symbol. Since the average transmit power with channel inversion power control policy on Rayleigh faded channel is infinite [11], a truncation threshold $g_{\mathrm{th}}^{2}$ is used in the channel inversion power control. This threshold $g_{\mathrm{th}}^{2}$ is set to a value such that a pre-specified transmission outage probability is met. The expression for the outage probability will be given in closed-form in Section III. In Step 3, the required power as well as the constellation parameter can be found numerically solving BER expressions of the hierarchical 4/16-QAM [12]. However, as mentioned above for analytical tractability, we use the approximations in Eq. (6).

\section{Comparison with Uniform QAM Constellation-based Scheme}

Multi-user scheduling is also possible using uniform QAM modulation in a time division fashion. The scheme is quite similar to the downlink multiplexing technique that we described in [6]. Basically, the whole transmission slot is divided into a number of mini-slots and in each mini-slot the data of a particular user is transmitted. In two user scheduling, the transmission slot is divided into two mini-slots. The first best user is scheduled in the first mini-slot whereas the second best user is scheduled in the second mini-slot. The transmit power in each mini-slot is adjusted according to the corresponding user's channel gain so that the bits are transmitted below the target BER using uniform 16-QAM over AWGN channel.

\section{Performance Analysis And Results}

In this Section, we derive analytical expressions for the average transmission power, the outage probability and the packet transmission probability of users for all the schemes under consideration. Numerical results obtained via computer simulations are also presented to compare the performance of the schemes under consideration. We use an uncoded target $\mathrm{BER}, \mathrm{BER}_{0}$ of $10^{-4}$ and a pre-specified outage probability of $10^{-3}$. We assume channel variation among the users is i.i.d with an average channel fading gain of $1 \mathrm{~dB}$.

\section{A. Outage Probability}

For two-user opportunistic scheduling a transmission outage occurs if the channel fading gain of the second best user, $g_{2}$ is less than a threshold $g_{\mathrm{th}}^{2}$. Therefore, the outage probability of two-user scheduling, $\mathrm{P}_{\text {out }}^{2}$ can be written as

$$
\mathrm{P}_{\text {out }}^{2}=\int_{g_{\mathrm{th}}^{2}}^{+\infty} \int_{g_{2}}^{+\infty} p_{g_{(1)} g_{(2)}}\left(g_{1}, g_{2}\right) d g_{1} d g_{2}
$$

where $p_{g_{(1)} g_{(2)}}\left(g_{1}, g_{2}\right)$ is the joint pdf of the largest two channel gains and can be expressed as [14]

$$
\begin{aligned}
p_{g_{(1)} g_{(2)}}\left(g_{1}, g_{2}\right)= & \frac{K(K-1)}{\left(g_{0}\right)^{2}} \mathrm{e}^{-g_{1} / g_{0}} \mathrm{e}^{-g_{2} / g_{0}} \\
& \times\left(1-\mathrm{e}^{-g_{2} / g_{0}}\right)^{K-2} .
\end{aligned}
$$

Using Eq. (9) we can the evaluate the integral in Eq. (8) as follows:

$$
\mathrm{P}_{\text {out }}^{2}=\sum_{i=1}^{K-2}\left(\begin{array}{c}
K-2 \\
i
\end{array}\right)(-1)^{i} \frac{K(K-1)}{i+1} \mathrm{e}^{-(i+1) g_{\mathrm{th}}^{2}} / g_{0} .
$$


For single-user scheduling a transmission outage occurs if the channel gain of the first best user is below a threshold $g_{\text {th }}^{1}$ and thus the outage probability of single-user scheduling, $\mathrm{P}_{\text {out }}^{1}$ can be written as

$$
\mathrm{P}_{\text {out }}^{1}=\int_{g_{\mathrm{th}}^{1}}^{+\infty} p_{g_{(1)}}\left(g_{1}\right) d g_{1}
$$

where $p_{g_{(1)}}\left(g_{1}\right)$ is the pdf of the largest channel gain and is known to be given by [14]

$$
p_{g_{(1)}}\left(g_{1}\right)=\frac{K}{g_{0}} \mathrm{e}^{-g_{1} / g_{0}}\left(1-\mathrm{e}^{-g_{1} / g_{0}}\right)^{K-1} .
$$

Using Eq. (12) in Eq. (11), the outage probability for single user scheduling can be written in desired closed-form as

$$
\mathrm{P}_{\mathrm{out}}^{1}=\sum_{i=1}^{K-1}\left(\begin{array}{c}
K-1 \\
i
\end{array}\right)(-1)^{i} \frac{K}{i+1} \mathrm{e}^{-(i+1) g_{\mathrm{th}}^{1}} / g_{0} .
$$

Solving Eqs. (10) and (13) for a given transmission outage, we can find the values of the thresholds $g_{\mathrm{th}}^{2}$ and $g_{\mathrm{th}}^{1}$, respectively.

\section{B. Average Transmit Power}

Averaging Eq. (6) over the channel gain joint pdf of the two best users, the average transmit power in two-user hierarchical constellation-based scheduling, $\mathrm{P}_{\mathrm{H}}^{2}$ can be expressed as

$$
\begin{aligned}
\mathrm{P}_{\mathrm{H}}^{2}= & \int_{g_{\mathrm{th}}^{2}}^{+\infty} \int_{g_{2}}^{+\infty}\left[\frac{C_{2}}{g_{1}}+\frac{C_{2}}{g_{1} g_{2}}\left(g_{1}+2 \sqrt{g_{1} g_{2}}+g_{2}\right)\right] \\
& \times p_{g_{(1)} g_{(2)}}\left(g_{1}, g_{2}\right) d g_{1} d g_{2}
\end{aligned}
$$

Substituting Eq. (9) in Eq. (14), the expression of $\mathrm{P}_{\mathrm{H}}^{2}$ can be writen as

$$
\begin{aligned}
\mathrm{P}_{\mathrm{H}}^{2}= & \frac{C_{2} K(K-1)}{\left(g_{0}\right)^{2}} \int_{g_{\mathrm{th}}^{2}}^{+\infty}\left[2 \int_{g_{2}}^{+\infty} \frac{\mathrm{e}^{-g_{1} / g_{0}}}{g_{1}} d g_{1}\right. \\
& \left.+\frac{2}{\sqrt{g_{2}}} \int_{g_{2}}^{+\infty} \frac{\mathrm{e}^{-g_{1} / g_{0}}}{\sqrt{g_{1}}} d g_{1}+\frac{1}{g_{2}} \int_{g_{2}}^{+\infty} \mathrm{e}^{-g_{1} / g_{0}} d g_{1}\right] \\
& \times \mathrm{e}^{-g_{2} / g_{0}}\left(1-\mathrm{e}^{-g_{2} / g_{0}}\right)^{K-2} d g_{2} .
\end{aligned}
$$

Similarly the average power in uniform 16-QAM constellation-based two user scheduling, $\mathrm{P}_{\mathrm{U}}^{2}$ can be written in simplified form as

$$
\begin{aligned}
\mathrm{P}_{\mathrm{U}}^{2}= & \frac{C_{4} K(K-1)}{2\left(g_{0}\right)^{2}} \int_{g_{\mathrm{th}}^{2}}^{+\infty}\left[\int_{g_{2}}^{+\infty} \frac{\mathrm{e}^{-g_{1} / g_{0}}}{g_{1}} d g_{1}+\frac{1}{g_{2}}\right. \\
& \left.\times \int_{g_{2}}^{+\infty} \mathrm{e}^{-g_{1} / g_{0}} d g_{1}\right] \mathrm{e}^{-g_{2} / g_{0}}\left(1-\mathrm{e}^{-g_{2} / g_{0}}\right)^{K-2} d g_{2}, \text { (16) }
\end{aligned}
$$

where the constant $C_{4}=2 N_{0} \Gamma\left(2^{4}-1\right)$. Unfortunately, the above integrals in Eqs. (15) and (16) can not be evaluated in closed-form. However, it can be evaluated numerically and consequently the average transmit power for two-user scheduling can be obtained.

The average transmit power for single-user scheduling, $\mathrm{P}^{1}$ can be expressed as

$$
\mathrm{P}^{1}=\int_{g_{\mathrm{th}}^{1}}^{+\infty} \frac{C}{g_{1}} p_{g_{(1)}}\left(g_{1}\right) d g_{1} .
$$

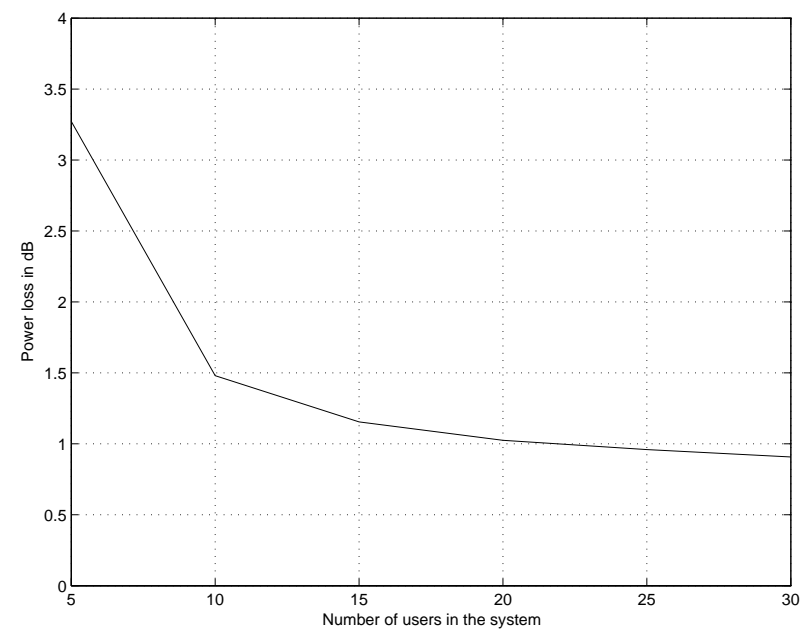

Fig. 2. Power loss of the hierarchical two best user scheduling with respect to the single user scheduling.

Substituting Eq. (12) in Eq. (17), $\mathrm{P}^{1}$ can be simplified to

$$
\mathrm{P}^{1}=\sum_{i=1}^{K-1} \frac{C_{4} K}{g_{0}}\left(\begin{array}{c}
K-1 \\
i
\end{array}\right)(-1)^{i} \operatorname{Ei}\left(a_{i}\right)
$$

where $a_{i}=\frac{(i+1) g_{\mathrm{th}}^{1}}{g_{0}}$ and $\operatorname{Ei}(\cdot)$ is an exponential integral function which is defined as

$$
\operatorname{Ei}(x)=\int_{1}^{+\infty} \frac{e^{-x t}}{t} d t
$$

The average power loss defined as the ratio of the average transmit power in single-user scheduling $\mathrm{P}^{1}$ to the average transmit power in two-user scheduling with hierarchical constellation, $\mathrm{P}_{\mathrm{H}}^{2}$ is plotted in Fig. 2. There are two observations from this figure. First, the two user scheduling always requires more power than the single user scheduling. This is due to the inclusion of the second best user in the transmission. Second, the required additional power decreases as the number of users in the system increases. This can be explained by the fact that as the number of users increases in the system the frequency of having higher difference between the first largest channel gain $g_{1}$ and the second largest channel gain $g_{2}$ decreases. Therefore, the average power required to transmit to the second best user decreases. The average power gain defined as the ratio of average transmit power in two-user scheduling with hierarchical constellation, $\mathrm{P}_{\mathrm{H}}^{2}$ to the average transmit power in two-user scheduling with uniform constellation, $\mathrm{P}_{\mathrm{U}}^{2}$ is plotted in Fig. 3. The gain in this figure is expected due to the superposition of the information bits for two different users in the same message symbol. It is also obvious from Fig. 3 that the gain decreases as the number of users increases in the system. This is again expected, as the frequency of having higher difference between $g_{1}$ and $g_{2}$ decreases with the number of users in the system. 


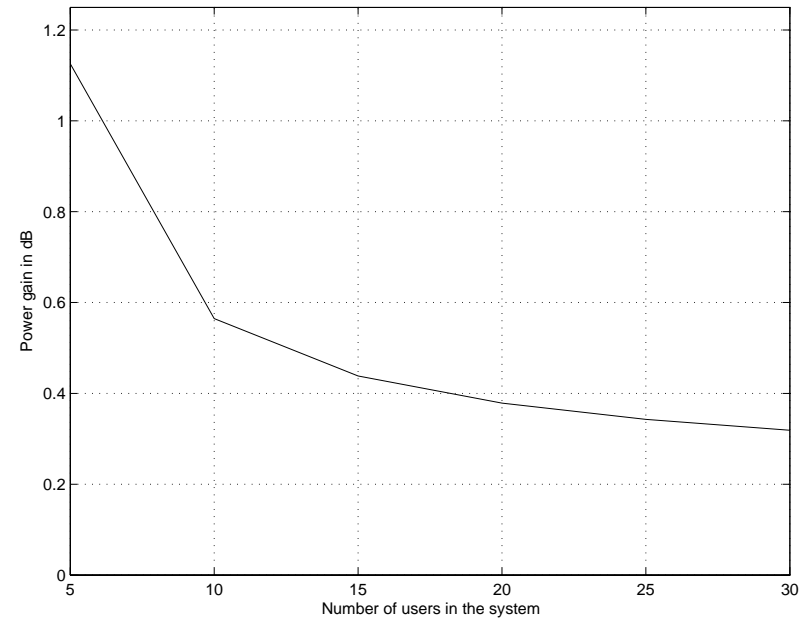

Fig. 3. Power gain of the hierarchical two best user scheduling with respect to the uniform constellation-based two user scheduling.

\section{Access Probability}

For i.i.d. channel variations among $K$ users, the probability that a user will get an access to channel is $2 / K$. Therefore, the probability of transmission by a user, for a pre-specified outage probability $P_{\text {out }}$, is expressed as

$$
\mathrm{P}_{\text {access }}^{2}=\frac{2}{K}\left(1-\mathrm{P}_{\text {out }}\right)
$$

Since the probability of selecting a given user out of $K$ users is $1 / K$ for single-user scheduling per time slot, the probability that a user will transmit a packet can be written as

$$
\mathrm{P}_{\text {access }}^{1}=\frac{1}{K}\left(1-\mathrm{P}_{\text {out }}\right) \text {. }
$$

The packet transmission probabilities, which plotted in Fig. 4 , show that the two-user scheduling (for both the hierarchical and mini-slotted schemes) offers double packet transmission probability compared to the classical single user scheduling. This is very obvious as the two-user scheduling transmits the data of two users in a given time slot.

\section{CONCLuSion}

In this paper, we proposed and studied a new hierarchical constellation-based multi-user opportunistic scheduling. The multi-user scheduling offers more frequent access to the channel and packet transmission at the expense of transmit power compared to the classical single user scheduling. The required additional power decreases as the number of users increases in the system. However, multi-user scheduling with hierarchical constellations require less transmit power than a uniform constellation-based mini-slotted scheme. As a future research topic we are interested to explore some upper layer performances, e.g., delay and packet loss rate of the multi-user opportunistic scheduling schemes.

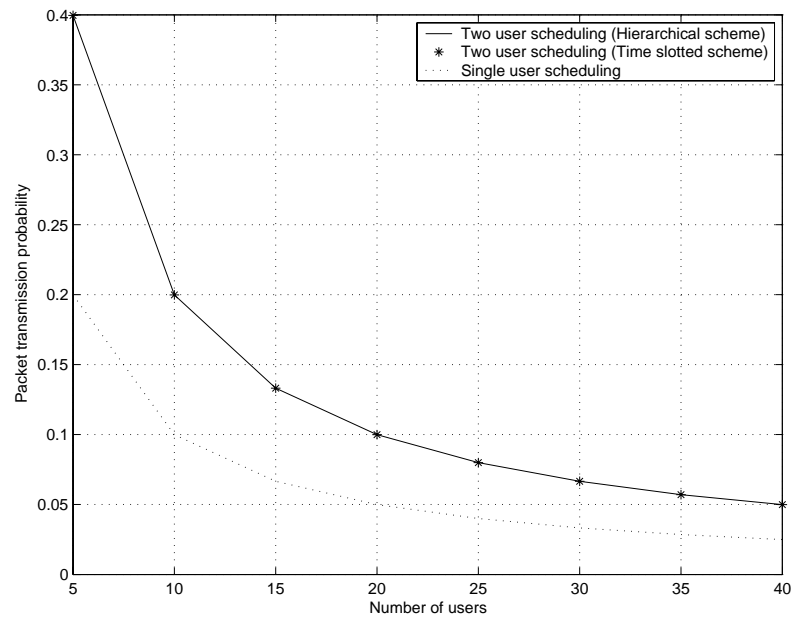

Fig. 4. Access probability versus number of users.

\section{REFERENCES}

[1] D. N. Tse, "Opportunistic beamforming using dumb antennas," IEEE Trans. Inform. Theory, vol. 48, pp. 1277-1294, June 2002.

[2] D. I. Kim, "Two-best user scheduling for high-speed downlink multicode CDMA with code constraint," in Proc. IEEE Conf. Global Commun. (Globecomm' 04), Dallas, TX, Oct. 2004, pp. 2659-2663.

[3] S. Dost, M. Oğuz Sunay, and V. K. Bhargava, "A feasibility study of two user downlink transmission for IS-856 system," in Proc. Personal and Indoor Commun. Conf. (PIMRC'04),, Bercelona, Spain, Sept. 2004, pp. 2029-2033.

[4] P. K. Vitthaladevuni and M.-S. Alouini, "A recursive algorithm for the exact BER computation of generalized hierarchical QAM constellations," IEEE Trans. Inform. Theory, vol. 49, no. 1, pp. 297-307, Jan. 2003.

[5] M. Morimoto, M. Okada, and S. Komaki, "A hierarchical image transmission system in fading channel," in Proc. IEEE Int. Conf. Univ. Personal Commun. (ICUPC' 95), Tokyo, Japan, October 1995, pp. 769772.

[6] Md. J. Hossain, P. K. Vitthaladevuni, M.-S. Alouini, and V. K. Bhargava, "Hierarchical modulations for multimedia and multicast transmission over fading channels," in Proc. IEEE Veh. Technol. Conf. (VTC'03 Spring), vol. 4, Jeju, Korea, April 2003, pp. 2633-2637.

[7] M. B. Pursley and J. M. Shea, "Adaptive nonuniform phase-shift-key modulation for multimedia traffic in wireless networks," IEEE Journal Select. Areas Commun., vol. 18, no. 8, pp. 1394-1407, August 2000.

[8] Md. J. Hossain, P. K. Vitthaladevuni, M.-S. Alouini, V. K. Bhargava, and A. J. Goldsmith, "Adaptive hierarchical constellations for simultaneous voice and multi-class data transmission over fading channels," in Proc. IEEE IV Workshop on Advances in Signal Process. for Wireless Commun. (SPAWC' 03), Rome, Italy, June 2003, Also accepted to be published in IEEE Trans. Veh. Technol.

[9] S. Pietrzyk and G. J. M. Janssen, "Subcarrier and power allocation for QoS-aware OFDMA systems using embedded modulation," in Proc. Int. Conf. Commun. (ICC' 04), vol. 6, Paris, France, June 2004, pp. $3202-$ 3206.

[10] T. Cover, "Broadcast channel," IEEE Trans. Inform. Theory, vol. IT-18, pp. 2-14, January 1972.

[11] A. J. Goldsmith, "The capacity of downlink fading channels using variable power and variable rate," IEEE Trans. Veh. Technol., vol. 46, no. 19, pp. 569-580, August 1997.

[12] P. K. Vitthaladevuni, and M.-S. Alouini, "BER computations of $4 / M$ QAM hierarchical constellations," IEEE Trans. Broadcasting, vol. 47, no. 3, pp. 228-239, September 2001. See also correction in IEEE Trans. Broadcasting, vol. 49, pp. 408, December 2003.

[13] J. M. Cioffi, "A multicarrier premier," November 1991, also available in http://www.stanford.edu/group/cioffi/pdf/multicarrier.pdf.

[14] T. Eng, N. Kong, and L. B. Milstein, "Selection combining schemes for rake receivers," in Proc. 4th Int. Conf. Univ. Personal Commun., Tokyo, Japan, November 1995, pp. 426-430. 